\title{
Considerazioni sulla conservazione di un paesaggio archeologico etrusco: il caso delle fortificazioni di Cerveteri
}

Considerations on the conservation of an etruscan archaeological landscape: the case of Cerveteri fortifications

\section{Tommaso Vagnarelli}

Politecnico di Torino, Turin, Italy, tommaso.vagnarelli@polito.it

\begin{abstract}
Where now stands the city of Cerveteri (RM), between the ninth and third century BC took form one of the most important city-states of Etruria: Caere. Today this place is especially noted for the presence of the Etruscan necropolis of Banditaccia, inserted in the UNESCO World Heritage List in 2004. The remains of the ancient fortifications, that represent one of the most interesting and well-preserved examples of an Etruscan defensive wall, are instead less known. Due to a prolonged abandonment and a presence of uncontrolled vegetation, the conservation of this testimony is now compromised by visible signs of deterioration. At the same time, this condition has defined a suggestive archaeological landscape in which architecture and nature coexist in a precarious balance. This contribution tries to deepen the importance of preserving this evocative symbiosis and proposes a possible conservative approach that could manage the relationship between ruins and vegetation.
\end{abstract}

Keywords: Landscape, ruins, conservation, Etruscans.

\section{Introduzione: l'Etruria immaginata}

Nel saggio Scienza e poesia alla scoperta dell'Etruria (Pallottino, 1957), pubblicato nel 1957 all'interno dei Quaderni dell'Associazione Culturale Italiana, Massimo Pallottino, padre dell'etruscologia contemporanea ${ }^{1}$, poneva l'accento su un interessante aspetto che fino a quel momento aveva caratterizzato il dibattito sulla materia etrusca: l'esistenza, cioè, di due distinte percezioni, per lo più contrastanti e inconciliabili tra loro, della civiltà etrusca. Vi era infatti, secondo quanto affermato da Pallottino, un'Etruria degli studiosi, della ricerca obiettiva, dell'analisi delle fonti e dell' archeologia, e un'Etruria dei letterati e dell'intuizione poetica, figlia della cultura del XIX secolo, fautrice di una tradizione di leggende moderne e di suggestioni romantiche e per lo più refrattaria a rettifiche di natura scientifica. In questa seconda Etruria ha preso forma, nel corso di più di due secoli, fino ai giorni nostri, il mito -e pregiudizio- che raffigura gli Etruschi quale popolo misterioso e indecifrabile: ancora oggi, infatti, problematiche storiche del dibattito estruscologico quali la lingua, la scrittura e le origini, benché in gran parte chiarite negli ultimi decenni, conservano intatto, agli occhi del vasto pubblico, il loro fascino oscuro e seducente, alimentando una visione di questa civiltà che procede per le vie traverse del fantastico e che risponde a verità proprie, in apparenza immuni al disincanto che la ricerca obiettiva spesso produce.

Tale fascinazione, che sarebbe affrettato giudicare con sufficienza come fenomeno di 
"ignoranza" popolare, nasce e si trasforma a partire da quelle esperienze letterarie che colsero ed espressero il sentimento di entusiastico stupore che attraversò l'Europa a partire dal Settecento ${ }^{2}$ quando, inaspettatamente, la Toscana e il Lazio dischiusero i propri tesori rivelando memorie di una civiltà che nessuno poteva immaginare tanto ricca, culturalmente complessa e artisticamente distante dalle forme dell'antichità classica a cui l'Occidente era stato fino ad allora abituato. Fu così che questi luoghi iniziarono ad attrarre antiquari, esploratori, scrittori e pittori e l'Etruria entrò a tutti gli effetti tra le tappe predilette degli itinerari archeologici del Grand Tour, al pari di Atene, Roma, Paestum e Agrigento. Da quel momento, attraverso opere come Tour to the Sepulchres of Etruria (1839) di Elizabeth Caroline Hamilton Gray, Cities and Cemeteries of Etruria (1848-1878) di George Dennis, L'antica Etruria marittima (1846) di Luigi Canina, i più recenti Etruscan Places (1932) di David Herbert Lawrence e Those Barren Leaves (1925) di Aldous Huxley e gli scritti di poeti come Giosuè Carducci e Gabriele D' Annunzio ${ }^{3}$, ebbe inizio la favola romantica dell'Etruria.

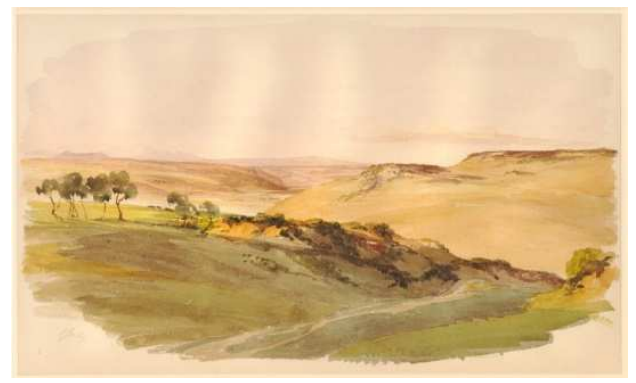

Fig. 1. Il paesaggio di Cerveteri in un acquerello di S. J. Ainsley (1842).

Alla radice di questo fenomeno non vi era, però, solo la meraviglia provata di fronte alle migliaia di vasi dipinti, gioielli, armi e statuette bronzee che emersero dai numerosi scavi condotti in quegli anni: ciò che più attraversò l'immaginario dell'epoca, in un momento fertile come non mai per suggestioni di questo tipo, fu il paesaggio (Fig. 1). Un paesaggio che, nel suo incarnare il sublime e il pittoresco romantico, dovette mostrarsi come dimora ideale per questa civiltà, dominato com'era da un susseguirsi ininterrotto di valli e altopiani, di campi coltivati e pascoli, di alture tufacee e forre boscose, di rovine e di centri abitati arroccati su alture scoscese, immersi in un'atmosfera che, meglio di qualsiasi altra, si prestava ad essere trasfigurata in realtà letteraria. Molti di questi luoghi, ancora oggi -e qui forse risiede la risposta al perdurare di tale fascinazione-, conservano in gran parte integri i propri caratteri paesaggistici originari, solo marginalmente intaccati dall'espandersi incontrollato dei centri urbani. È questo il caso delle vaste aree archeologiche che si sviluppano intorno ai centri di Populonia, Tarquinia, Vulci, Cerveteri, nelle quali i monumenti e la natura si fondono in una suggestiva sintesi che è qualità peculiare di molti contesti etruschi.

Un esempio emblematico di questa simbiosi è rappresentato dai resti delle mura difensive della Cerveteri di epoca etrusca, una testimonianza di grande rilievo archeologico e architettonico, immersa in una dimensione ambientale unica. Per lo stato di abbandono in cui questo monumento versa da anni e per l'interesse da sempre marginale suscitato anche nella trattatistica più specializzata, si intende qui di seguito presentarne un breve approfondimento, nella duplice volontà di evidenziarne le qualità storiche, architettoniche e paesaggistiche e di suggerire una possibile strategia di conservazione che sia capace di garantire la sopravvivenza non solo del manufatto in sé, ma anche di quei rapporti di coralità (Pane, 1987, p. 302) che legano inscindibilmente le antiche testimonianze al loro ambiente naturale.

\section{Cerveteri: la città e l'archeologia}

Analizzando il caso studio in oggetto, è innanzitutto interessante notare come proprio Cerveteri, conosciuta anticamente con il nome di Caere, rappresenti uno degli esempi forse più significativi della dicotomia evidenziata da Pallottino: da un lato, infatti, lo studio della vastissima area archeologica che oggi circonda la città ha rappresentato uno dei momenti fondamentali per gli studi etruscologici dell'ultimo secolo, continuando tuttora ad essere luogo privilegiato per la ricerca, dall'altra il suo contesto, le sue necropoli e i resti della città antica sono stati più volte protagonisti di pagine 
letterarie fondamentali per la definizione dei confini poetici dell'Etruria; basti ricordare, per esempio, le descrizioni memorabili e cariche di sentimento che D. H. Lawrence dedicò ai paesaggi funerari di Cerveteri all'interno del suo Etruscan Places (Lawrence, 1932, pp. 29-45).

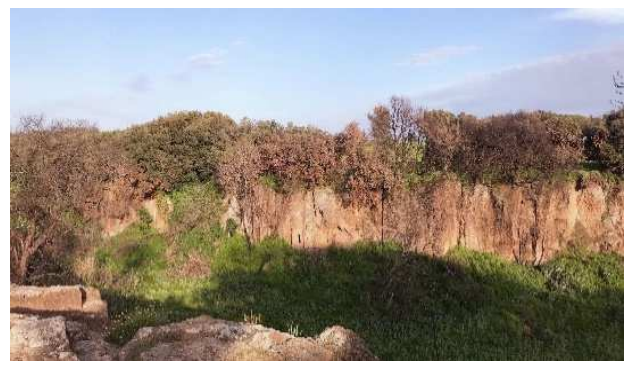

Fig. 2. Il pianoro dei Vignali e il fosso del Manganello visti dalla Banditaccia.

Nota anche con il nome etrusco Cisra e con quelli

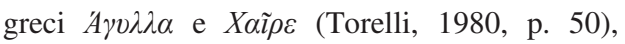
l'antica Caere sorse a circa $6 \mathrm{~km}$ dal mare, sviluppandosi al di sopra di tre pianori tufacei paralleli, di forma allungata, separati tra loro da profonde gole scavate dai torrenti del Manganello e della Mola. Sull'altura centrale, nota come pianoro dei Vignali (Fig. 2), di circa centocinquanta ettari di superficie e delimitata su tre lati da alte scogliere di tufo, prese forma l'abitato storico, mentre le altre due, a nord-ovest e sud-est della città, chiamate Banditaccia e Monte Abatone, vennero destinate alle omonime necropoli monumentali (Proietti, 1986, p. 17). Tra il VII e durante tutto il VI secolo a.C., all'apice del periodo di espansione etrusca sul mare, Caere fu una città ricca e fiorente, centro di un regno indipendente capace di influenzare, se non politicamente, certo culturalmente, l'intera Etruria e di intrattenere commerci con molti tra i popoli che abitavano il Mediterraneo. Oggi Cerveteri è conosciuta soprattutto per le sue necropoli, una parte delle quali è entrata nel 2004 nella World Heritage List dell'UNESCO ${ }^{4}$; meno note al pubblico, solo parzialmente indagate e non valorizzate, benché ricadenti anch'esse nel perimetro del sito UNESCO e ugualmente tutelate ai sensi del Codice dei Beni Culturali e del Paesaggio (Dlgs 42/2004), sono invece le tracce superstiti della città, quali ponti, porte urbiche, opere di ingegneria idraulica e resti di fortificazioni. Escluse dalle operazioni di risistemazione che dagli anni Dieci del Novecento interessarono la parte più monumentale del sepolcreto (Porretta, 2018), oggi unico settore dell'area archeologica ad essere custodito ed attrezzato per la visita, queste testimonianze, che costellano in gran numero il territorio circostante, vennero a lungo dimenticate e condannate a un decennale abbandono, perpetuatosi fino ai giorni nostri. Tale condizione, se da un lato ha determinato l'insorgere di fenomeni di deterioramento anche gravi, legati in gran parte alla crescita incontrollata della vegetazione, dall'altra ha definito i contorni di un paesaggio archeologico unico, che oggi appare più che mai importante preservare: un luogo di grande suggestione in cui l'elemento artificiale e quello naturale coesistono senza apparente soluzione di continuità, in cui il contesto che accoglie i manufatti ne diventa esso stesso parte integrante.

\section{Le mura etrusche di Caere}

Tra le aree esterne al sito di visita ${ }^{5}$, di grande rilievo, per la quantità e la varietà di testimonianze archeologiche presenti, oltre che per i caratteri unici del paesaggio, è il tratto perimetrale nordoccidentale del pianoro dei Vignali, nel punto in cui il costone tufaceo che definiva il perimetro e le difese naturali dell'antica città, diminuendo gradualmente di livello, rende più agevole l'attraversamento del fosso del Manganello e la salita verso l'antistante altopiano della Banditaccia.

Quest'area liminare, di confine tra la "città dei vivi e la città dei morti", in cui i sepolcri si spingono fino ai piedi dell'abitato, è forse il luogo più significativo per comprendere lo stretto rapporto che intercorreva tra queste due realtà, strette in un dialogo fisico, visivo e simbolico non inconsueto nella concezione distributiva degli insediamenti etruschi (Bartoloni, 2016, p. 92).

Era infatti qui che, tramite tre porte urbiche ancora oggi chiaramente distinguibili, le cosiddette porte della Via degli Inferi, della Bufolareccia e Coperta, gli antichi Ceriti attraversavano il peri- 


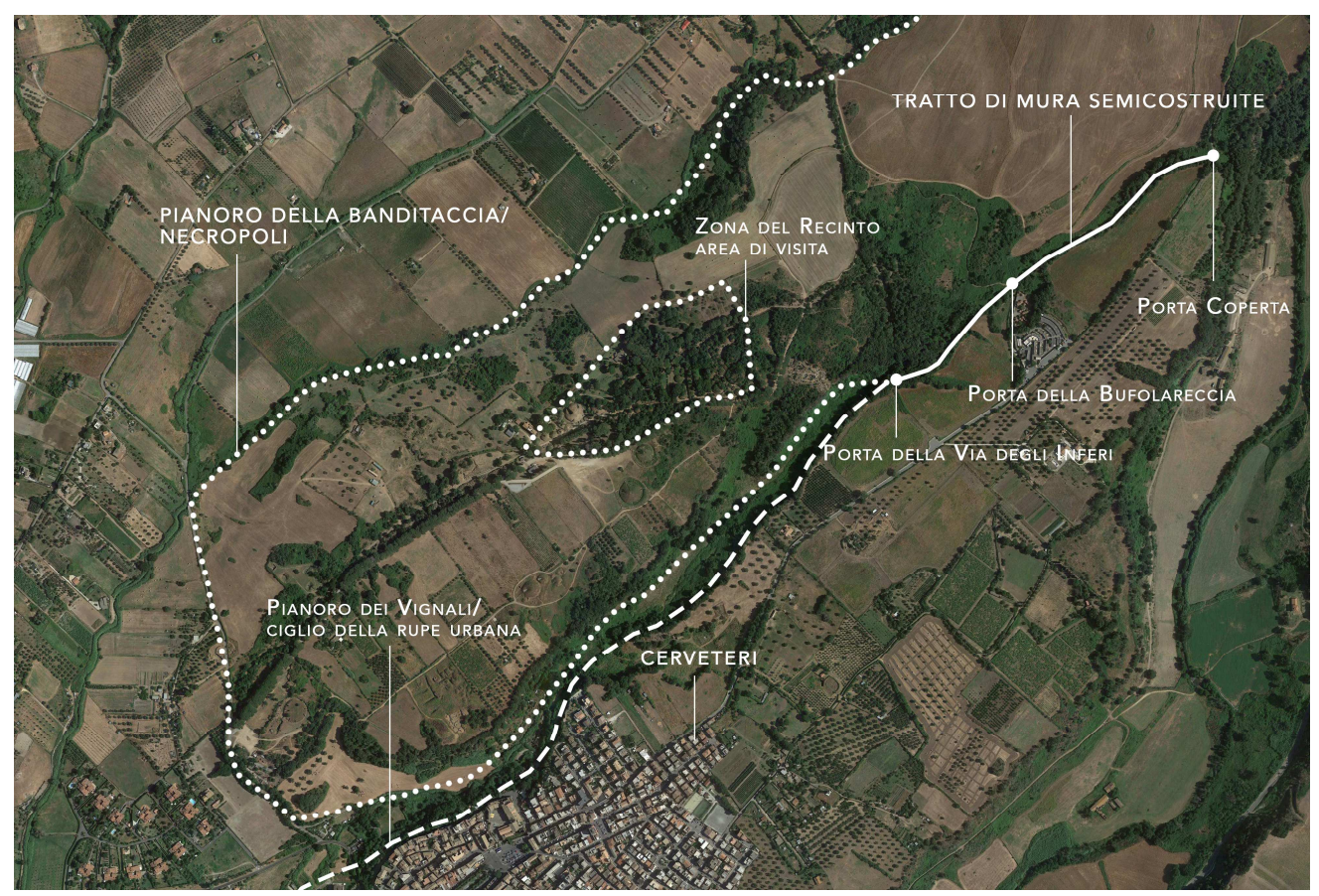

Fig. 3. Ortofoto con evidenziati i confini del pianoro dei Vignali e della Banditaccia, le mura e le porte urbiche.

metro del centro abitato e accedevano alla necropoli, percorrendo vie che, al contempo, proseguivano verosimilmente al di là dei confini del sepolcreto, connettendo Caere alla porzione settentrionale del suo territorio e al mare (Fig. 3).

Chi giungeva alla città da nord, o chi vi si allontanava, era quindi vincolato da percorsi che si dipanavano attraverso una distesa sterminata di tombe monumentali, simboli della ricchezza materiale e culturale della città. I segni di questa articolata realtà sono oggi ancora chiaramente leggibili e concorrono a definire il contorno di un mosaico archeologico e culturale eterogeneo e stratificato. L'elemento che più caratterizza questo settore dell'area archeologica è il tratto di mura semi-costruite, lungo circa $800 \mathrm{~m}$, compreso tra i resti della porta della Via degli Inferi e della Porta Coperta e parte del più ampio sistema difensivo naturale che cingeva l'intera città per circa $7 \mathrm{~km}$.

Qui, le pendici dell'altopiano, che nella porzione compresa tra le due porte urbiche diminuivano notevolmente d'altezza a causa dell'orografia della valle del Manganello, subirono, tra il VI e il
V secolo a.C., nell'ambito di un più vasto progetto di monumentalizzazione dell'intero perimetro cittadino, un duplice, massiccio, intervento volto a colmare con fortificazioni artificiali le carenze difensive della rupe urbana (Bellelli, 2014, pp. 50-53). Da un lato, il fronte nordoccidentale del pianoro, in quel tratto forse poco più di un declivio, venne verticalizzato attraverso un'imponente opera di scavo che consentì di aumentare l'altezza della parete e di creare un profondo fossato con fronti antistanti alti anche più di $10 \mathrm{~m}$; dall'altra, contemporaneamente, l'orlo superiore del pianoro venne regolarizzato e alzato di livello grazie alla realizzazione di un muro difensivo in opera quadrata quasi regolare, composto da blocchi parallelepipedi disposti per testa ${ }^{5}$, e rinforzato da un terrapieno retrostante (Fig. 4).

Dove la roccia presentava discontinuità non superabili tramite regolarizzazione, gli stessi blocchi vennero utilizzati a guisa di risarcitura del fronte difensivo, in una sorta di vera e propria operazione scuci e cuci. Nelle ipotesi ricostruttive ottocentesche di autori come Luigi Canina questa cortina muraria si supponeva cingesse tutto il pianoro, elevandosi per diversi metri sopra il ciglio 

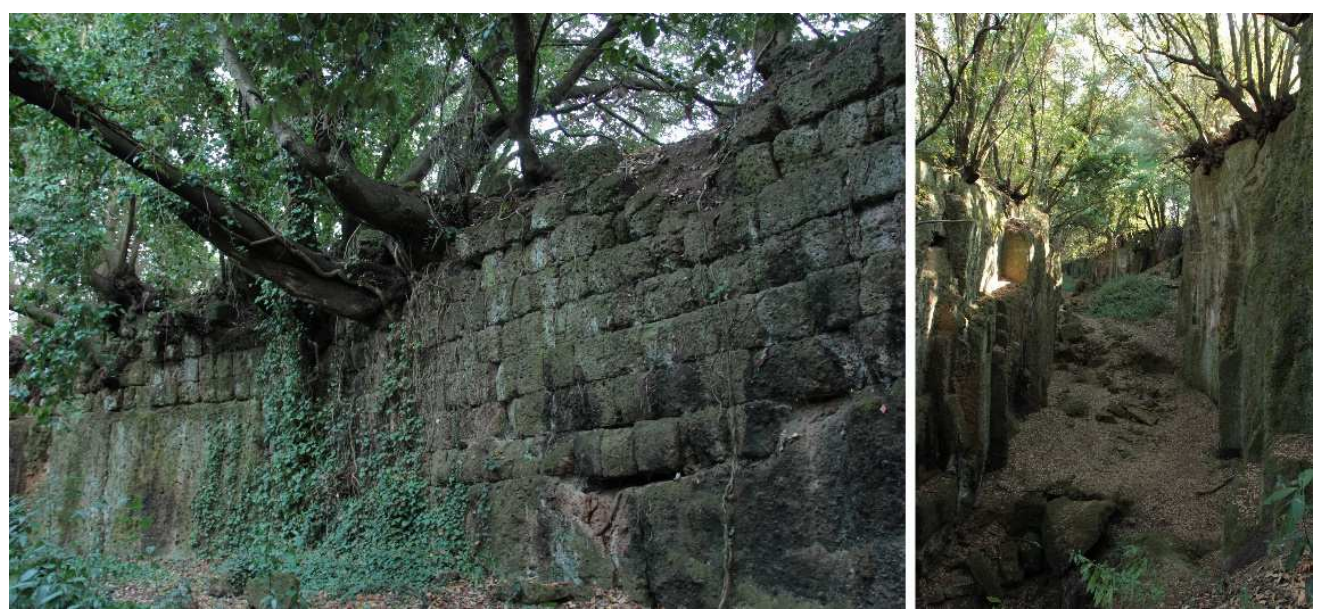

Fig. 4. A sinistra le mura semicostruite, a destra il fossato nel tratto verticalizzato.

dell'altopiano. Non vi sono, ad oggi, abbastanza dati per confutare queste ipotesi, ma, a seguito delle più recenti indagini archeologiche -le ultime, in ordine di tempo, sono quelle condotte nella zona delle mura tra il 2010 e il 2011 (Bellelli, 2014, p. 50)-, si tende a considerare come più attendibile la versione proposta dal Lugli, secondo cui Caere ebbe mura solo laddove necessario, nei punti in cui la rupe non garantiva un'adeguata protezione (Lugli, 1957, p. 273).

Questa concezione del costruire, che si risolveva e trovava efficacia proprio nella sintesi funzionale tra natura e costruito, è una caratteristica ridondante nell'architettura etrusca, riscontrabile non solo nelle fortificazioni, ma anche negli abitati e, soprattutto, in ambito funerario ${ }^{7}$. Furono le maestranze di Caere, per prime, a partire dal VII secolo a.C., ad affinare e a diffondere questa tecnica costruttiva, che qui toccò vette di raffinatezza altrove insuperate (Colonna, 1986, pp. 394-398).

\section{Ruderi, vegetazione, memoria e conservazione $e^{8}$}

Non è avventato supporre che quelli che oggi appaiono quali caratteri peculiari del paesaggio archeologico dell'Etruria possano derivare, in parte, proprio da questa vocazione terrigena al costruire, da questo intimo legame che l'architettura etrusca riuscì sempre ad intessere con la natura circostante. Tale concezione fu, sì, dettata soprattutto da necessità pratiche, ma non si può escludere celasse anche implicazioni più profonde, connesse a motivazioni estetiche e simboliche: è infatti evidente come nel suo incarnare le forme di un'Antichità altra, distante dalla maniera proporzionata ed euritmica della coeva Grecia, e nel suo essere così immediata, vivace, a tratti espressionista (Morolli, 1985, pp. 31-33), l'architettura etrusca sembri mostrare una congenita inclinazione al pittoresco, radicata com'è al contesto dal quale emerge. Una simile morfologia può far supporre che già in antichità i monumenti funebri, così come le mura cittadine, tendessero inevitabilmente ad amalgamarsi con la vegetazione circostante; se questa integrazione fosse però ricercata e apprezzata, se le edere, i muschi e i licheni che anche ai giorni nostri ne ricoprono i resti fossero, all'epoca, frutto di un deliberato intento ornamentale, ci è ignoto.

Ciò che invece si conosce con certezza è che già dal principio dell'Ottocento, agli occhi dei primi esploratori romantici che attraversavano le campagne d'Etruria, la vegetazione si manifestò quale costante di questi luoghi, quale elemento di raccordo tra ruderi e natura, metafora del genius loci che per millenni aveva abitato queste terre. P roprio a causa della vegetazione, il tratto fortificato oggetto di questa disanima è stato per molti decenni pressoché celato alla vista.

Solo negli ultimi anni, grazie principalmente all'attività costante delle associazioni di volontari 


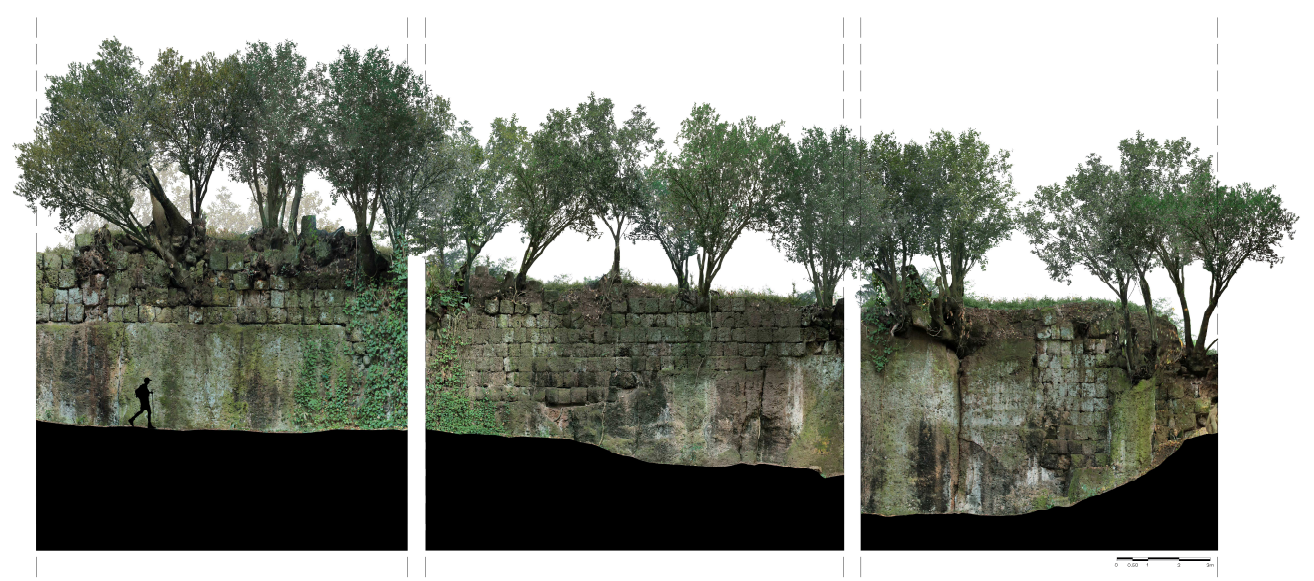

Fig. 5. Fotopiano delle mura difensive nel tratto semicostruito (rilievo ed elaborazione di T. Vagnarelli).

che si occupano di ripulire l'area, il sentiero che corre lungo il perimetro delle mura e che collega tra loro i resti delle principali porte urbiche è tornato percorribile e le fortificazioni, almeno inparte, visibili. Nulla ha però impedito alla vegetazione di svilupparsi incontrollata al di sopra del manufatto, determinando numerose situazioni di rischio per la conservazione delle strutture e provocando, nei casi più gravi, lesioni e crolli parziali. Tuttavia, questa lenta aggressione, questo graduale processo che assimila l'architettura all'opera della natura, ha contribuito a definire la potenza evocativa di questo luogo, che oggi appare regolato da dinamiche tanto affascinanti, quanto complesse e conflittuali. Come si può notare dai rilievi presentati in queste pagine (Fig. 5), la situazione odierna in cui versano le mura appare particolarmente articolata: oltre alla presenza di vegetazione inferiore, quali muschi, licheni e funghi, di vegetazione arbustiva, come edera e rovi, e di erbacee di vario tipo, lungo il ciglio superiore della rupe fortificata si è sviluppata una fitta cortina di alberi ad alto fusto. $\mathrm{Si}$ tratta di lecci, querce, bagolari e fichi, le cui radici, sfruttando plessi fessurativi preesistenti nel materiale litico e punti di più bassa resistenza come i giunti tra i blocchi di tufo, sono penetrate nell'orditura muraria, avviluppandosi alle strutture e provocando distacchi di materiali e fessurazioni. Alcune di queste piante appaiono pericolosamente protese verso l'esterno e una loro eventuale caduta, tutt'altro che improbabile, provocherebbe il crollo di importanti porzioni di muro.
In un contesto simile, la volontà di salvaguardare l'integrità non solo materica, ma anche paesaggistica, del bene aggiunge un ulteriore grado di complessità al progetto di conservazione, che potrà risolversi efficacemente solo tramite la pianificazione di adeguati interventi di restauro e di costanti pratiche manutentive che prevengano gli effetti più negativi dell'entropia verso cui la componente naturale muove costantemente.

La complessità di operare in una tale condizione risiede proprio nel determinare quali siano le azioni corrette da intraprendere per mantenere questo delicato equilibrio, mediando tra la necessità, e la priorità, di conservare le architetture e la scelta di non rimuovere gran parte della vegetazione, azione che altererebbe non solo la qualità estetica di questo luogo, ma soprattutto la percezione della memoria storica e del valore di antichità di cui questo contesto si fa portatore e la cui massima espressione si rivela nel rapporto stesso, indissolubile, tra ruderi e natura (Romeo, Morezzi, Rudiero, 2017, p. 32).

A tal fine, tra le possibili pratiche attuabili, potrebbe innanzitutto risultare efficace redigere un rilievo floristico che permetta di catalogare le specie vegetali presenti nell'area archeologica e di valutarne, tramite lo studio delle caratteristiche biologiche, il grado di interferenza con il manufatto stesso (Signorini, 1996, pp. 7-14). Tale operazione, affiancata da indagini diagnostiche che analizzino il rapporto tra quadro fessurativo delle strutture, dissesti e posizione delle radici delle 
piante, consentirebbe di definire strategie di selezione e di controllo della flora che possano ridurre ai soli casi strettamente necessari l'intervento di rimozione degli alberi cresciuti sulle murature. Benché una simile azione possa parzialmente ledere la simbiosi estetica tra costruito e ambiente, vi sono diversi casi in cui si renderebbe necessaria, soprattutto in quelle situazioni in cui sono già in atto evidenti meccanismi di degrado.

La procedura di asportazione della vegetazione arborea, laddove inevitabile, andrebbe sempre effettuata con estrema cautela, seguendo una metodologia specifica: è infatti necessario, dopo aver devitalizzato completamente la pianta, predisporre interventi sistematici sul manufatto, sia prima della rimozione, con preconsolidamenti e opere provvisionali di sostegno che contrastino eventuali movimenti o cedimenti della struttura, sia dopo, con consolidamenti e reintegrazioni che colmino le eventuali fessurazioni preesistenti e i vuoti lasciati dall'asportazione delle alberature stesse (Musso, 2013, p. 46). In presenza di aree monumentali di grande valore paesaggistico è facile intuire come l'approccio più cauto e corretto sia quello di limitare tali operazioni solo ai casi in cui vi siano rischi concreti per i ruderi. Dove si rilevassero, invece, condizioni potenzialmente dannose, ma non al punto da necessitare di soluzioni così drastiche, bisognerebbe preferire una costante pratica manutentiva, che possa monitorare le interazioni tra la vegetazione e il costruito e gestire le dinamiche evolutive che ne caratterizzano la convivenza (Mancini, Rossi Doria, 2017, pp. 33-41). Nel caso delle mura di Caere, in cui la vegetazione arborea ha avuto modo di assumere, indisturbata, proporzioni in certi casi eccessive, incompatibili con la salvaguardia delle strutture, sembrerebbe più immediato e semplice optare per la prassi operativa più consueta, cioè la totale eliminazione dell'elemento biodeteriogeno.

Il dato archeologico sarebbe così preservato, ma la desertificazione del monumento, l'impermeabilizzazione dello stesso al contatto con la natura, tradirebbe il senso profondo dell'orizzonte etrusco e di un paesaggio che è esso stesso monumento, ricco di memorie dell'uomo che proprio nella fusione con la natura trovano la loro dimensione di sigillo del tempo sulla materia.
Solo se le strategie di conservazione sapranno rispondere a questa sfida, accettando la complessità di un intervento che non si limiti al monumento, ma sappia, piuttosto, governare le trasformazioni di un intero contesto e le forze opposite che vi abitano, l'Etruria immaginata, quella del Dennis e del Lawrence e delle poesie di Carducci, potrà continuare ad essere evocata nel reale: essa si cela infatti nella dimensione del conflitto, lì dove le volontà contrastanti dell'uomo e della natura si oppongono in equilibrio perfetto.

\section{Note}

${ }^{1}$ Massimo Pallottino (1909-1995) è stato storico del mondo antico, archeologo ed etruscologo. Fu il primo docente di etruscologia alla Sapienza di Roma (1937-1940).

${ }^{2}$ A questo secolo risalgono i primi scavi archeologici in contesti etruschi, precisamente a Cortona, Volterra e Tarquinia (Proietti, 1986, p. 20).

${ }^{3} \mathrm{Ci}$ si riferisce alla poesia Avanti! Avanti! (Carducci, 1882) e al romanzo Forse che sì, forse che no (D’Annunzio, 1910).

${ }^{4}$ La nomina del sito a Patrimonio UNESCO è dovuta alla presenza di centinaia di tombe in ottimo stato di conservazione, che attraversano circa cinque secoli di storia etrusca. Il loro studio è stato fondamentale per comprensione di molti aspetti della società etrusca, con particolare riferimento alla cultura funeraria, all'arte e all' architettura.

5 Il perimetro di visita della Banditaccia, si estende su una superficie di circa 15 ettari. Il resto dell'area archeologica a settentrione della città, equivalente alla zona occupata dal pianoro della Banditaccia e da parte di quello dei Vignali, si estende per oltre 200 ettari.

${ }^{6}$ Da misurazioni effettuate in situ, i blocchi risultano di dimensioni più o meno regolari: circa 45 $50 \mathrm{~cm}$ di altezza e larghezza, circa 90 di lunghezza.

${ }^{7}$ Sono numerosi gli esempi di architetture etrusche che uniscono all'intaglio dei banchi tufacei naturali integrazioni in opera quadrata o di altro tipo: i casi più noti sono quelli delle tombe a tumulo e a dado della Banditaccia, in cui questo 
schema appare quasi ovunque rispettato. Si veda Colonna, 1986.

${ }^{8}$ Sul tema dell' architettura allo stato di rudere e della conservazione dei paesaggi archeologici si faccia riferimento alle ricerche di Emanuele Romeo ed Emanuele Morezzi, in particolare: Romeo, Morezzi, 2019; Romeo, Morezzi, Rudiero, 2017.

\section{Bibliography}

Bartoloni, G. (2016). Introduzione all'Etruscologia, Hoepli, Milano.

Bellelli, V. (2014). "Caere e Pyrgi: il territorio, la viabilità e le fortificazioni”, in Atti della giornata di studio (Roma, Consiglio Nazionale delle Ricerche, $1^{\circ}$ marzo 2012), Caere 6, Pisa-Roma.

Canina, L. (1846-1851). L'antica Etruria marittima, compresa nella dizione pontificia, descritta ed illustrata con $i$ monumenti, Roma.

Carducci, G. (1882). Giambi ed Epodi, Bologna

Colonna, G. (1986). "Urbanistica e Architettura", in Pallottino, M.; Torelli, M.; Cristofani, M.; Camporeali, G.; Colonna, G.; Roncalli, F.; Mansuelli G.A.; Bonghi Jovino, M.; Simone, C. De, Rasenna, storia e civiltà degli Etruschi, UTET, Torino.

D’Annunzio, G. (1910). Forse che sì, forse che no, Calman-Lévi, Parigi.

Dennis, G. (1883). The Cities and Cemeteries of Etruria, John Murray, Londra.

Lawrence, D.H. (1932). Etruscan Places, Londra.

Lugli, G. (1957). La tecnica edilizia romana con particolare riferimento a Roma e Lazio, Bardi, Roma.

Mancini, R.; Rossi Doria, I. (2017). Ruderi e Vegetazione, questioni di restauro, Ginevra Bentivoglio Editoria, Roma.

Morolli, G. (1985). Vetus Etruria. Il mito degli Etruschi nella letteratura architettonica, nell'arte e nella cultura da Vitruvio a Winckelmann, Alinea, Firenze.

Musso, S.F. (2013). Tecniche di Restauro - aggiornamento, UTET, Torino.

Pallottino, M. (1957). Scienza e poesia alla scoperta dell'Etruria, Quaderni ACI, 24.

Pane, P. (1987). "C. G. Jung e i due poli della psiche”, in Pane, P., Attualità e dialettica del restauro: educazione all'arte, teoria della conservazione e del restauro dei monumenti, M. Solfanelli Ed., Chieti.

Porretta, P. (2018). L'invenzione moderna del paesaggio antico della Banditaccia. Raniero Mengarelli a Cerveteri, Edizioni Quasar, Roma.

Proietti, G. (1986). Cerveteri, Edizioni Quasar, Roma.

Romeo, E.; Morezzi, E. (2019). Che almeno ne resti il ricordo. Memoria, evocazione, conservazione dei beni architettonici e paesaggistici, Aracne, Roma.

Romeo, E.; Morezzi, E.; Rudiero, R. (2017). Riflessioni sulla conservazione del patrimonio archeologico, Aracne, Roma.

Signorini, M.A. (1996). L'Indice di Pericolosità: un contributo del botanico al controllo della vegetazione infestante nelle aree monumentali, Inf. Bot. Ital., 28 (1).

Torelli, M. (1980). Etruria, Laterza, Bari. 